\title{
Profiling neuronal epigenomes in a mouse model of alcohol use disorder
}

\author{
C.R. Bridges, O. Ponomareva, C.T. Tulisiak, T. Larina, R.A. Harris, I. Ponomarev* \\ Waggoner Center for Alcohol and Addiction Research, The University of Texas, Austin, Texas, USA \\ *e-mail:ponomarev@utexas.edu
}

Key words: alcoholism, neuroscience, epigenomics, INTACT, ATAC-Seq

Motivation and Aim: Alcohol use disorder (AUD) is characterized by widespread changes in gene expression in humans and animal models. Mounting evidence points to a central role of chromatin (epigenomic) modifications in controlling gene expression and behavior in AUD, but there is a critical gap in our knowledge regarding AUD-associated epigenomic changes in specific neuronal populations in the addiction neurocircuits. Here, we tested two recently developed methods: INTACT (Isolation of Nuclei TAgged in specific Cell Types) and ATAC-Seq (Assay for Transposase-Accessible Chromatin using Sequencing) to obtain epigenomic profiles from limited number of cells [1]. INTACT is based on the Cre-loxP system in mice to express a tagged nuclear membrane protein, allowing affinity purification of tagged nuclei from genetically defined cell populations. ATAC-Seq provides a comprehensive description of the epigenomic state in the cell, including chromatin accessibility (open chromatin), nucleosome positioning and occupancy of DNA binding proteins. The aim of this study was to examine the effects of alcohol on the epigenome of glutamatergic neurons in the prefrontal cortex, a key structure with a role in the preoccupation/anticipation stage of AUD.

Methods and Algorithms: Alcohol dependence was induced by ethanol vapor inhalation [2] in genetically modified mice expressing green fluorescent protein (GFP) in Camk2a-positive cells. Twenty four hours after ethanol was removed brains were dissected for molecular profiling. We used INTACT to isolate nuclei from glutamatergic cortical neurons and ATAC-Seq to obtain genome-wide distributions of open chromatin marks.

Results: We achieved $>95 \%$ specificity and up to $70 \%$ yield of GFP + neurons. We tested ATAC-Seq using various numbers of nuclei and were able to obtain reliable epigenomic profiles from as few as 5,000 nuclei. Statistical and bioinformatics analysis are underway to identify genes and gene networks affected by alcohol.

Conclusion: Identifying alcohol-induced, cell type-specific molecular changes is critical to our understanding of the roles of individual neuronal populations in alcohol actions, and a combination of INTACT and ATAC-Seq will be instrumental in studying neuronal epigenomes in a brain region - and neural circuit - specific manner.

Acknowledgements: Supported by NIAAA grants AA024586 and Bruce/Jones Graduate Fellowship in Addiction Biology.

\section{References}

1. Mo A. et al. (2015) Epigenomic signatures of neuronal diversity in the mammalian brain. Neuron. $86: 1369-1384$.

2. Lopez M.F., Becker H.C. (2005) Effect of pattern and number of chronic ethanol exposures on subsequent voluntary ethanol intake in C57BL/6J mice. Psychopharmacol. 181:688-696. 